\title{
TUNNEL MODELING USING MOBILE MAPPING LIDAR POINTS
}

\author{
S. S. Deshpande \\ Surveying Engineering, Penn State University, Wilkes-Barre, USA, ssd5269@psu.edu
}

Commission VI, WG VI/4

KEY WORDS: Tunnel, Mobile Mapping, lidar

\begin{abstract}
:
In this paper, a method to model a tunnel using lidar points is presented. The data used was collected using Leica Pegasus Two Ultimate with a Z+F 9012 Profiler mounted on a mobile platform. The tunnel was approximately $151 \mathrm{~m}$ long. Visual inspection of a cross-section of the tunnel showed two rail tracks supported on ballast and sidewalks along both sidewalls of the tunnel. The walls and the ceiling of the tunnel were made of five planar surfaces. The tunnel alignment was straight, without any horizontal or vertical curves. The bearing of the central axis of the tunnel was N12.2oW. The following methodology was developed to model just the planar surfaces of the tunnel by excluding the rails, ballast, sidewalks, powerlines, and other accessories.

The entire methodology was divided into three broad parts. In the first part, a model cross-section was created. Since the design plans of the tunnel were not available, the model cross-section polyline was created using mean tunnel dimensions from random cross-section points. The model cross-section consisted of the walls and the ceiling of the tunnel. Points were placed at every $1 \mathrm{~cm}$ along the model polyline. Six of the model points that represented the shape of the tunnel were selected as salient points. The lower-left salient point was considered as the seed point. In the second part, to define a reference axis of the tunnel, an approximate centerline was manually defined by selecting points at its start and end. Lidar points within $1 \mathrm{~m}$ at the start and the end of the tunnel were modeled using the model points to determine the centroids. The reference axis was determined by connecting the centroids at the start and the end of the tunnel. In the third part, the tunnel points were sliced along the reference axis at $5 \mathrm{~cm}$ intervals. The model cross-section was matched to points within each tunnel slice using a three-stage approach. In the first stage, the pattern of salient points was matched to the tunnel points by placing the seed point at every tunnel point location. The distances between salient points and their nearest tunnel points were calculated. Ten sets of tunnel points with the least differences to the salient points were shortlisted. In the second stage, a dense point-to-point matching was performed between the model and sliced tunnel data at the shortlisted points. The shortlisted point location with the least difference between the tunnel and the model points was considered as a match. At this point, the model points were hinged to the tunnel points at the seed point location. Hence, in the last stage, a six-parameter affine transformation was performed to match the model points to the tunnel data. The transformed model points at every $5 \mathrm{~cm}$ of the length of the tunnel were considered as current shape of the tunnel.
\end{abstract}

\section{INTRODUCTION}

Transportation networks are arteries of any nation. Maintaining transportation infrastructure effectively is essential for the smooth functioning of any nation. Periodic monitoring of transportation infrastructure using traditional surveying techniques and instruments would require a significant amount of time and human efforts. Moreover, such methods could be used at sample locations thus may not provide details to greater resolutions.

Over the past decades, lidar technology has been increasingly being used to model transportation structures such as bridges and tunnels. Terrestrial scanners have substantially increased the quantity of data that can be collected. Similarly, a Mobile Mapping System (MMS) has also developed into a mature technology allowing the acquisition of 3D data on a very large scale. It consists of high-precision lidar (Light Detection and Ranging) sensors for data collection with GPS and Inertial Measurement Units (IMU) mounted on a mobile platform such as a trail or a car. Periodic monitoring can be achieved by adopting such newer and faster technology.

The quantity of data acquired using a lidar sensor is significantly high. Manual extraction of pertinent information from the data can be both time consuming and influenced by the operator's judgement. Therefore, faster and automated tools are essential to process the data effectively. In this paper, lidar data acquired in a railway tunnel is analysed to is increasingly used in transportation applications.

\subsection{Organization of the paper}

Section two of this paper summarizes recent pertinent publications in the field of lidar technology, particularly tunnel modelling. Section three describes the data used in this research. Section four explains the methodology developed to model tunnel using lidar points. Section five shows the results and discusses the findings. Section six describes the conclusions of the proposed methodology. This section also makes recommendations for future research topics.

\section{LITERATURE REVIEW}

Over the past decades, lidar sensors have developed into a rapid data acquisition technology. Lidar is based on the principle of distance measurement using light pulses. The time taken by the pulses from the sensor to the object is used to determine the range distance. Using the onboard GPS/IMU (Global Positioning System/Inertial Measurement Unit) system the 3D position of the point is determined (Liu et al., 2009). Lidar is being used on terrestrial (Lindenbergh et al., 2005, Han et al., 2013, Van Gosliga et al., 2006, Walton et al., 2014, Han et al., 2013), 
mobile (Tan et al., 2016, Boavida et al., 2012), and aerial platforms to collect points on the area of interest. Current terrestrial lidar systems can collect 100,000 to a million points per second.

Aerial lidar has been widely used in numerous studies in shoreline mapping (Li et al., 2008, Deshpande and Yilmaz, 2017), transportation engineering (Luo et al., 2012), and many more fields (Desh pande, 2013). Mobile Mapping System utilizes multiple sensors including lidar to acquire 3D points from a mobile platform. Various investigations to map assets and geometric properties of roads (Luo et al., 2012) and railways have been reported over the past several years. Several researchers have modeled tunnels using mobile and terrestrial lidar points (Gikas, 2012, Han et al., 2013). Research to model a circular or elliptical shaped tunnel has been more commonly reported (Nuttens et al., 2014, Kang et al., 2014, Argüelles-Fraga et al., 2013, Cheng et al., 2016, Arastounia, 2016, Yi et al., 2019). Researchers (Arastounia, 2016) have investigated as-built creation of circular/elliptical shaped tunnel by fitting an ellipse equation to the data at $5 \mathrm{~cm}$ intervals. This approach was suitable for circular or elliptical tunnel shape.

The problem of matching two data sets is a common matching problem in 3D image analysis. A representation-independent method for registering a variety of 3D shapes such as point sets, free-form curves, and surfaces was proposed in (Besl and McKay, 1992). Iterative closest point (ICP) matching algorithm is a versatile technique that has been used in numerous studies to align 2D or 3D points. Researchers (Liu et al., 2009) have registered 3D shapes using an Iterative Closest Point (ICP) algorithm as the priori knowledge of the correspondence between the points was not necessary.

In this research, since template and tunnel points were oriented in one plane, a new approach was developed to match these two sets of points. The actual centroidal axis is determined by computing the geometric mean of the cross-section shape of the tunnel. Various terminologies used in this research are described in the next paragraph.

\subsection{Terms used in tunnel modeling}

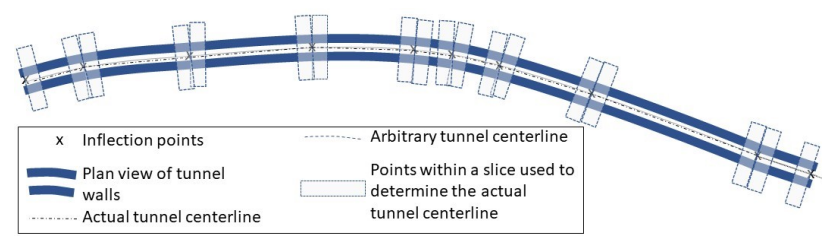

Figure 1. Schematic plan view of a tunnel.

Figure 1 shows a schematic diagram of a tunnel with multiple horizontal curves. The inflection points identified the locations of change in the horizontal alignment of the tunnel. The arbitrary centreline was created by connecting the inflection points. Starting from one end of the tunnel, points within slices on both sides of the inflection points along the arbitrary centreline were used to determine their geometric centres.

Figure 2 shows the lidar points within a $0.5 \mathrm{~m}$ width along the centreline. Only the wall points were used to determine the geometric centre. The actual centreline was then defined as a 3D line created by connecting these geometric centres of the slices. The process to determine the actual centreline is explained later in the paper.

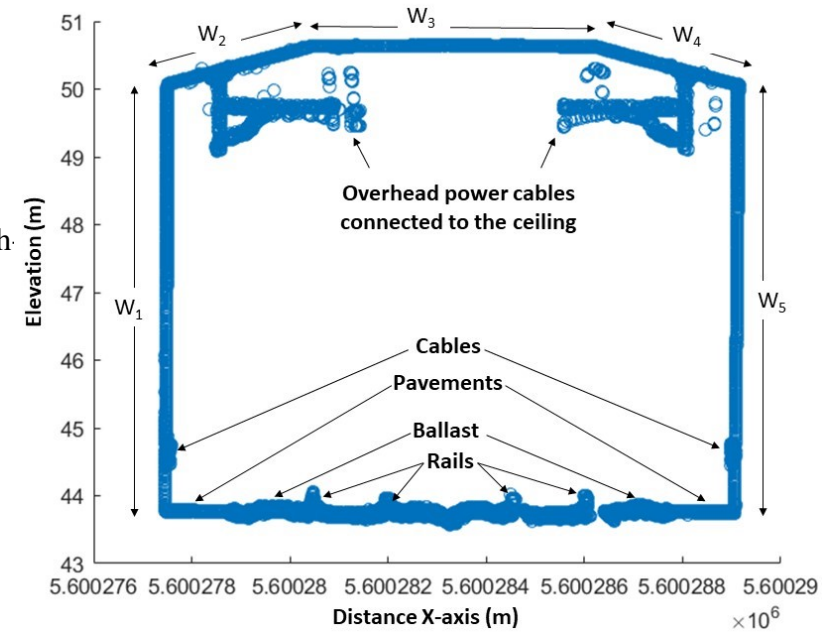

Figure 2. Cross-section of the tunnel showing various runnle elements.

\section{DESCRIPTION OF DATA USED}

The tunnel data used in this paper was acquired in August 2019 using Leica Pegasus Two Ultimate with a Z+F 9012 Profiler mounted on a mobile platform. Figure 3(a) shows the sketch of the undisclosed location of the tunnel that was built under-water channels. The tunnel was oriented approximately N12 ${ }_{o}$ W. Figure 3 (b) shows the point cloud of the tunnel and the nearby area. The point spacing of the data was $25 \mathrm{~mm}$. The tunnel data as shown in Figure 3 (c) was clipped to be used in this study. The length of the tunnel was approximately $151 \mathrm{~m}$.

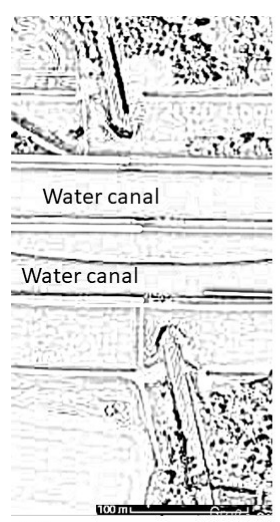

(a)

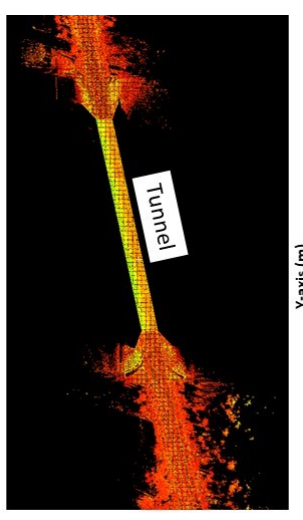

(b)

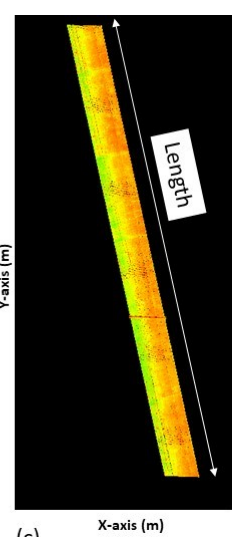

(c) $\quad$-axis (m)
Figure 3. Aerial view of the location. (a) Sketch of the location showing the water canals and the tunnel, (b) lidar points of the tunnel and nearby areas, and (c) clipped tunnel lidar points.

A cross-sectional view of the tunnel is shown in Figure 2. The tunnel was made of five planar surfaces (annotated as $W_{1}$ to $\left.W_{5}\right)$ and carried two rail lines that were laid on the ballast. Pavements were located along both walls of the tunnel. As seen in Figure 2, lidar points were also recorded on the two rail tracks, power line, power cables, and other accessories. The nominal width of the tunnel perpendicular to the centreline was $11.6 \mathrm{~m}$ and the nominal height from the pavement to the underside of the ceiling was $6.8 \mathrm{~m}$. 


\section{METHODOLOGY}

The entire methodology can be broadly divided as creating template cross-section points, locating the actual centreline of the tunnel, fitting tunnel template points to the lidar points at every cross-section perpendicular to the actual centreline, and analysis of deformation. Figure 4 shows an overview of the methodology.

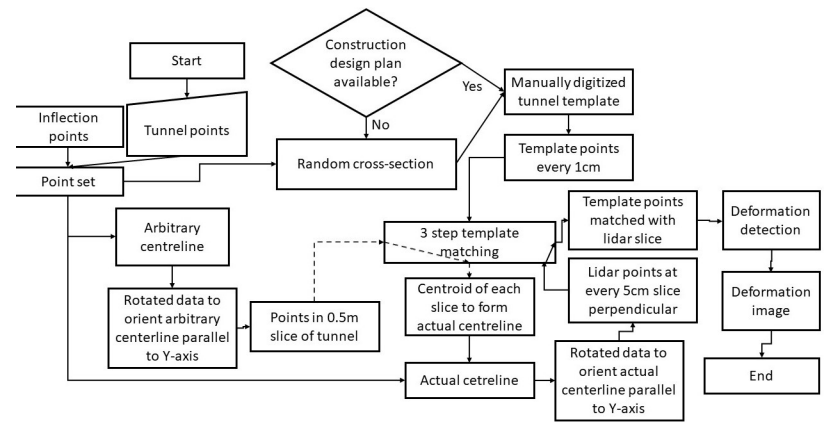

Figure 4. Workflow of the methodology.

\subsection{Tunnel cross-section template creation}

The actual construction drawings were not available for the tunnel used in this study. Hence, a slice of data was randomly selected and digitized manually to create a cross-section template. Figure 5 (a) shows lidar points in a $0.5 \mathrm{~m}$ wide cross-section. As an ideal tunnel design would have the walls as planar surfaces, the walls of the cross-section template were digitized as a polyline made of five segments as shown in Figure 5 (a). Features such as pavements, rails, ballasts, powerlines, and other accessories were not included in the cross-section template polyline.

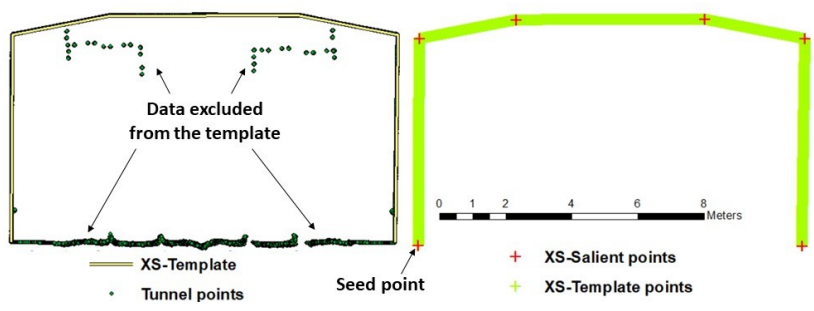

Figure 5. A tunnel cross- section template creation. (a) Points on the tunnel surface and the lines representing the tunnel walls and (b) template points placed at regular interval, salient points, and seed point.

Template points were placed at every $1 \mathrm{~cm}$ along the template polyline and six salient points were identified as shown in Figure 5 (b). At $1 \mathrm{~cm}$ spacing, the template was represented by 2430 points. The lower left salient point was considered as a seed point. The template, salient, and seed points were used in the matching process explained later in this paper.

\subsection{Actual Centreline Determination}

The actual centreline determination process is explained using a smaller segment of the tunnel data. Figure 6 (a) shows the plan view of $20 \mathrm{~m}$ length of the tunnel. The arbitrary centreline was created by connecting two manually selected inflection points as shown. The data was rotated to orient the arbitrary axis parallel to the Y-axis so that the sliced data points were parallel to
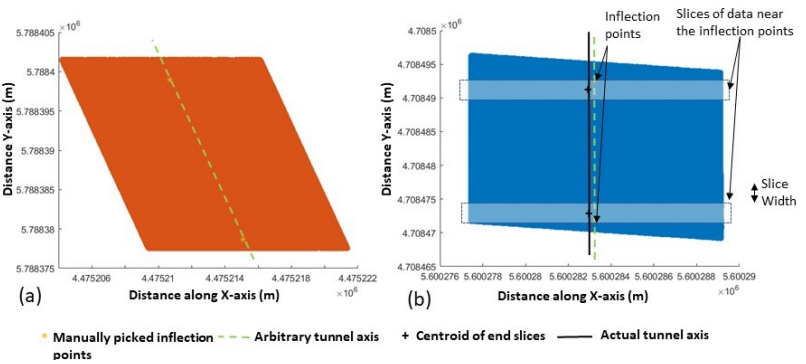

Figure 6. Plan view of the tunnel. (a) The approximate tunnel axis identified by two points and (b) Data rotated about the $\mathrm{Z}$-axis to orient the approximate tunnel axis parallel to the Y-axis.

the template points. Figure 6 (b) shows the two slices at the inflection points.

\subsection{Fitting template points to the sliced data}

Due to manual identification of the arbitrary centreline, the tunnel's axis was almost parallel to the Y-axis thereby orienting the points almost parallel to the template points. A wider slice of data would eliminate the effect of the small tilt as its centroid would be calculated using a larger lidar point set. Therefore, a wider slice of $0.5 \mathrm{~m}$ was used at the inflection points. At this point, it should be noted that 2430 template points and more than 110,000 tunnel points need to be spatially matched. Additionally, all points other than on the tunnel wall were to be excluded. To expedite the process, a three-stepped point-to-point matching method was developed to align the template points to the tunnel points. In the first step, the pattern of salient points was compared to the tunnel points. As state in Paragraph 4.1, the lower-left corner salient point was considered as a seed point that was compared at each tunnel point. The other salient points were translated with reference to the seed point and the closest tunnel point was determined to calculate the total separation between the two sets of points.

Difference $=\sum_{i=1}^{i=N}$ DistancefromS $P_{i}$ tonearesttunnelpoint

Where $S P$ are salient points. Tunnel point locations that matched the spatial pattern of the salient points were shortlisted as shown in Figure 6(a). The points with lowest ten cumulative distances were considered candidates for the next step.

In the second step, the shortlisted locations were checked to identify the best matching location. In this step, separations between all template points to their nearest tunnel point at each shortlisted location were calculated. The shortlisted location with the smallest separation was considered as a match. Figure 7 (b) shows the template points matched at the best location on the tunnel points. It should be noted that this match was hinged to the seed salient point position. To filter the points on the ground and railway accessories, tunnel points within $1 \mathrm{~cm}$ from the template points were only considered.

Additionally, the tunnel walls may not be perfectly orthogonal. Hence, in the third step a six-parameter affine transformation was implemented to best fit the template points to the tunnel points. The equation used is specified below:

$a_{1} x_{t}+a_{2} z_{t}+a_{3}=x_{d}$ 

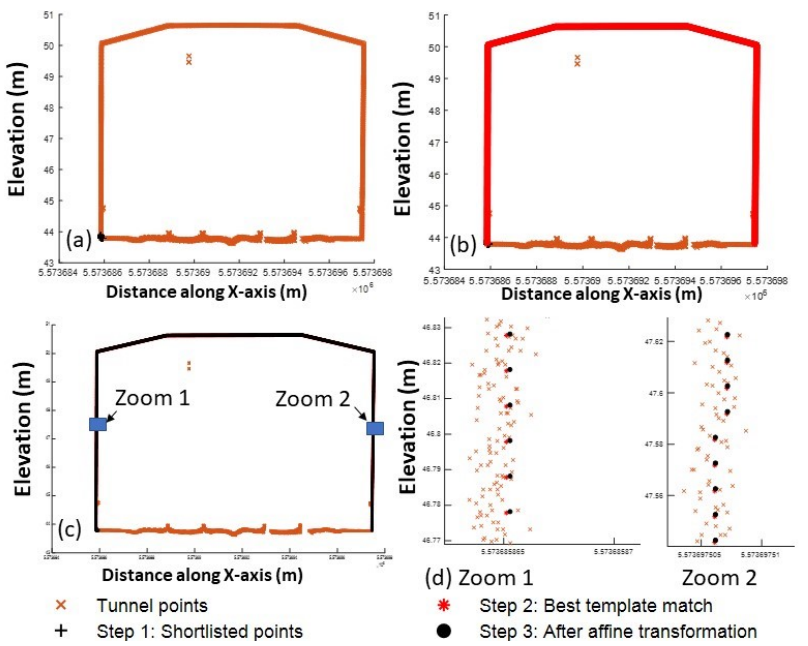

Figure 7. Step one of the point-to-point matching process to determine the actual centreline. (a) lidar points within a $0.5 \mathrm{~m}$ width along the arbitrary centreline, (b) template points oriented over the best matching location, (c) template points after the affine transformation, and (d) zoom-in view of the points.

$a_{4} x_{t}+a_{5} z_{t}+a_{6}=z_{d}$

Where,

- $x_{t}$ and $z_{t}$ are template points coordinates,

- $x_{d}$ and $z_{d}$ are nearest tunnel points coordinates, and

- $a_{1}$ to $a_{6}$ are the affine transformation parameters.

Similar to the previous step, to filter the points on the ground and railway accessories, tunnel points within $1 \mathrm{~cm}$ from the template points were only considered. The solution was calculated by least-squares adjustment.

At this point, the template points were oriented to the tunnel points. Due to the use of the affine transformation, the template points were rotated, scaled, and translated to best match the tunnel points. As specified earlier, points greater than $1 \mathrm{~cm}$ away from the template points were excluded. The centroids of the transformed template points were considered as the centroids of the slices. The actual centreline was obtained by connecting these centroids. The data was one more time rotated so that the actual centreline was parallel to the Y-axis. This step ensured that the cross-sections were perpendicular to this centreline and the tunnel cross-section points were oriented parallel to the template points.

\subsection{Slicing the data perpendicular to the central axis}

At this stage, the complete tunnel data along with the actual centreline of the tunnel was oriented parallel to the Y-axis. Starting at the southern inflection point, the lidar points were sliced perpendicular to the actual centreline and were fitted to the template points using the three-step matching process explained in Paragraph 4.3.

It should be noted that the template points were aligned to the tunnel points at every $5 \mathrm{~cm}$ distance of the tunnel. Points significantly away from the template points were excluded in the matching process. The distance between the template point to its nearest tunnel point was calculated. Considering the centre of the tunnel, if this was inside or outside the template points region was determined. The results obtained by this procedure are presented in the next section.

\section{RESULTS AND DISCUSSIONS}

The methodology explained in the previous section was implemented on the data shown in Figure 3. As the tunnel was straight without any curves, two inflection points were placed at the start and end of the tunnel to define the arbitrary centreline. The complete data was rotated to orient the arbitrary centreline parallel to the Y-axis. Lidar points within $0.5 \mathrm{~m}$ width at the inflection points (as described in Figure 5 (b)) were matched with the template points. The centroids of these two slices of data were used to define the actual centreline of the tunnel. The data was rotated one again to orient the actual centreline parallel to the Y-axis as shown in Figure 8 (b).

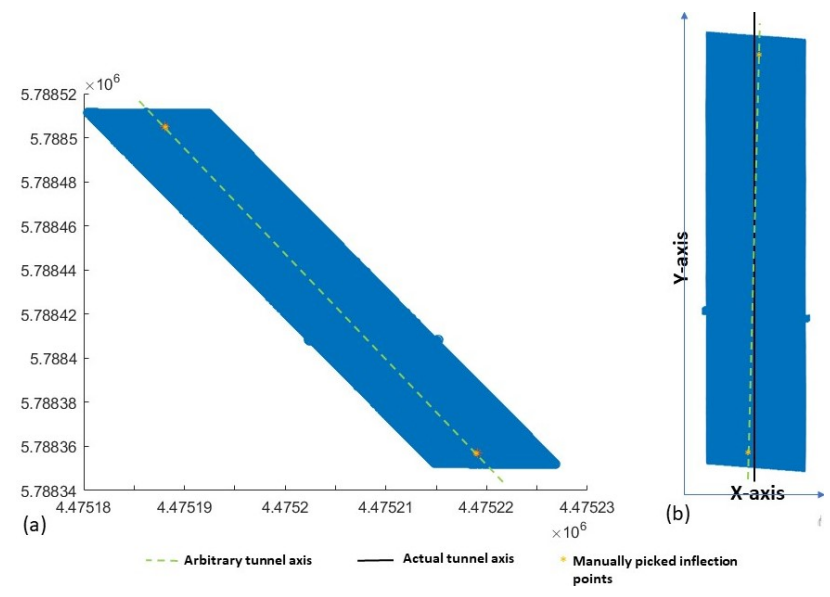

Figure 8. Plan view of the tunnel points. (a) Original orientation of the points, two inflection points, and the arbitrary centreline and (b) points after second rotation showing both the arbitrary and actual cerntreline.

This step ensured that the tunnel cross-sections were parallel to the template points in the $\mathrm{X}-\mathrm{Z}$ reference frame. Lidar points were sliced at every $5 \mathrm{~cm}$ interval. On average, 4850 points were located within each slice. The three-step matching process as described in Paragraph 4.3 was implemented to match the template points to the lidar points of each slice.

An example of lidar points within $5 \mathrm{~cm}$ width is shown in Figure 9 (a). After implementing the first step, the shortlisted points were identified. As shown in Figure 9 (b) the template points were placed at the point having lowest totat difference. Finally, the template points were transformed using affine model as shown in Figure 9 (c). This figure also showed the effectiveness of the method to exclude the non-ground points. A zoomed-in view of the points at two locations are shown in Figure $9(d)$.

Since the expected match in the next cross-section would be within a small extent of the match at the first location, the first step of matching was modified after the first cross-section. Instead of matching the salient point pattern at each point, only the points within $10 \mathrm{~cm}$ from the previously matched location in the $\mathrm{X}$ and $\mathrm{Z}$-axis were searched. This process further expedited the matching process. 

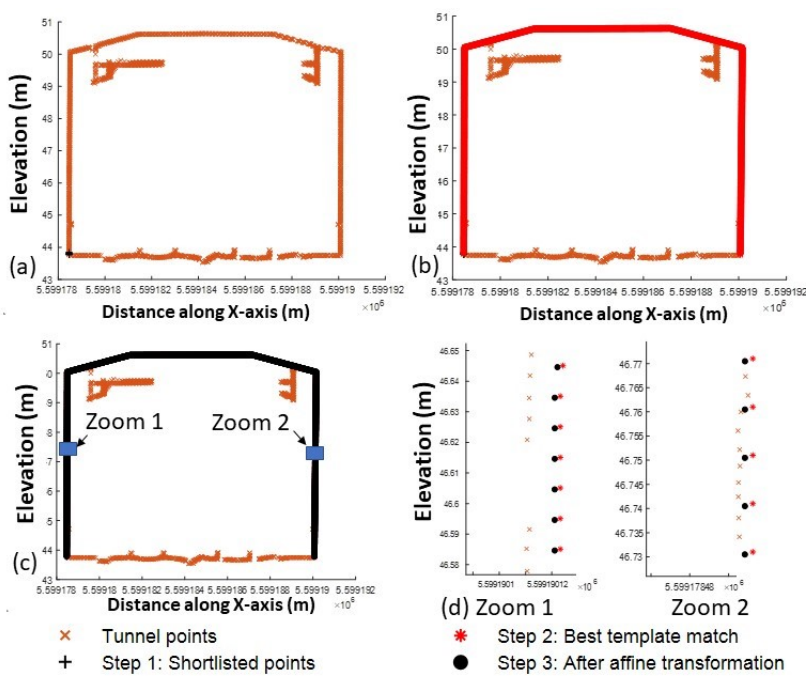

Figure 9. Example of template point matching. (a) Lidar points within a $5 \mathrm{~cm}$ slice of tunnel data perpendicular to the actual centreline, (b) Template matched at the best location. Outlier were filtered, (c) Points after affine transformation, and (d) zoomed-in view at two locations.

\subsection{Deformation detection}

The entire data was divided into 3025 slices extending $5 \mathrm{~cm}$ each from the southern inflection point to the northern. At this point, the template points were fitted to each slice. As stated earlier, the template consisted of 2430 points. The distances from every template point to the nearest tunnel point were calculated as deformations. The transformed template points were used as a reference to determine if the tunnel deformations were outward (positive deformations) or inward (negative deformations).

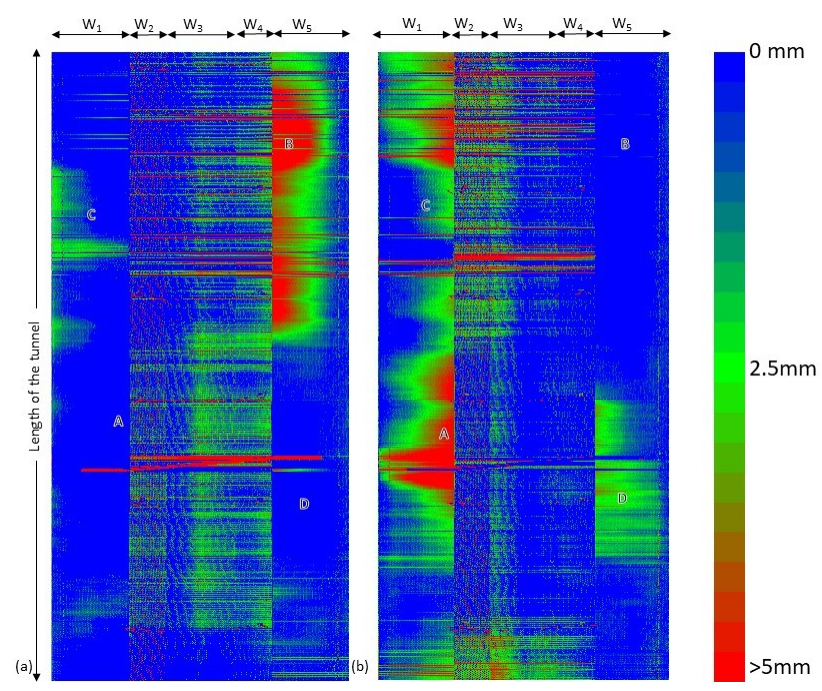

Figure 10. Tunnel deformation images. (a) Positive deformations and (b) Negative deformations

Images were created using the deformations recorded at each template point. The template points were organized as rows to form a deformation image where the column number corresponded to tunnel cross-sections. Figure 10 (a) and (b) show the positive and negative deformations, respectively. Each of these images consisted of 2430 columns and 3025 rows. The various wall segments: $W_{1}$ to $W_{5}$ were identified as shown in the figures. Both images were colour coded from blue (representing zero deformation) to red (greater than equal to $5 \mathrm{~mm}$ deformation). By comparing both these images, several deformations can be noted. If a region is shown in blue colour on both the images then that region has zero deformation. On the contrary, regions $\mathrm{A}$ and $\mathrm{D}$ show inward deformations. Region $\mathrm{C}$ shows that wall segment $W_{1}$ with positive deformations followed by negative deformations. Region B shows positive deformations. Horizontal stripes at locations were observed in the $W_{2}, W_{3}$, and $W_{4}$ segments. A visual check was performed that showed higher points in those regions.

\section{CONCLUSIONS}

This paper presented a method to model a tunnel using mobile mapping lidar points. An arbitrary centreline was manually defined to determine the actual centreline. Template points were matched to lidar points at every $5 \mathrm{~cm}$ distance along the tunnel's actual centreline using a three-step approach. Deformations in the tunnel surface were calculated with reference to the actual centreline. The results showed that this method was effectively able to model the tunnel. The template used in this research was not a regular shape such as a circle or ellipse. Therefore, the proposed method could be used to model tunnel of any shape.

While experimenting future research topics were discovered. In the current study, an arbitrary centreline was manually defined. Future work could focus on developing methodology to completely automate the process of defining the arbitrary centreline. Additionally, different types of transformation to match the template points to the tunnel points could be experimented to determine their impacts.

\section{ACKNOWLEDGEMENTS}

We would like to thank Leica Geosystem for providing the data.

\section{REFERENCES}

Arastounia, M., 2016. Automated as-built model generation of subway tunnels from mobile LiDAR data. Sensors, 16(9), 1486.

Argüelles-Fraga, R., Ordóñez, C., García-Cortés, S., RocaPardiñas, J., 2013. Measurement planning for circular crosssection tunnels using terrestrial laser scanning. Automation in construction, 31, 1-9.

Besl, P. J., McKay, N. D., 1992. Method for registration of 3-d shapes. Sensor fusion IV: control paradigms and data structures, 1611, International Society for Optics and Photonics, 586-606.

Boavida, J., Oliveira, A., Santos, B., 2012. Precise long tunnel survey using the riegl vmx-250 mobile laser scanning system. Proceedings of the 2012 RIEGL International Airborne and Mobile User Conference, Orlando, FL, USA, 27.

Cheng, Y.-J., Qiu, W., Lei, J., 2016. Automatic extraction of tunnel lining cross-sections from terrestrial laser scanning point clouds. Sensors, 16(10), 1648.

Deshpande, S. S., 2013. Improved floodplain delineation method using high-density LiDAR data. Computer-Aided Civil and Infrastructure Engineering, 28(1), 68-79. 
Deshpande, S., Yilmaz, A., 2017. A semi-automated method to create a lidar-based hydro-flattened DEM. International journal of remote sensing, 38(5), 1365-1387.

Gikas, V., 2012. Three-dimensional laser scanning for geometry documentation and construction management of highway tunnels during excavation. Sensors, 12(8), 11249-11270.

Han, S., Cho, H., Kim, S., Jung, J., Heo, J., 2013. Automated and efficient method for extraction of tunnel cross sections using terrestrial laser scanned data. Journal of computing in civil engineering, 27(3), 274-281.

Kang, Z., Zhang, L., Tuo, L., Wang, B., Chen, J., 2014. Continuous extraction of subway tunnel cross sections based on terrestrial point clouds. Remote Sensing, 6(1), 857-879.

Li, R., Deshpande, S., Niu, X., Zhou, F., Di, K., Wu, B., 2008. Geometric integration of aerial and high-resolution satellite imagery and application in shoreline mapping. Marine geodesy, 31(3), 143-159.

Lindenbergh, R., Pfeifer, N., Rabbani, T., 2005. Accuracy analysis of the leica hds 3000 and feasibility of tunnel deformation monitoring. Proceedings of the ISPRS Workshop, Laser scanning, 36, 3 .

Liu, J.-K., Li, R., Deshpande, S., Niu, X., Shih, T.-Y., 2009. Estimation of blufflines using topographic LiDAR data and orthoimages. Photogrammetric Engineering \& Remote Sensing, 75(1), 69-79.

Luo, X., Ren, X. T., Li, Y., Wang, J. J., 2012. Mobile surveying system for road assets monitoring and management. 2012 7th IEEE Conference on Industrial Electronics and Applications (ICIEA), IEEE, 1688-1693.

Nuttens, T., Stal, C., De Backer, H., Schotte, K., Van Bogaert, P., De Wulf, A., 2014. Methodology for the ovalization monitoring of newly built circular train tunnels based on laser scanning: Liefkenshoek Rail Link (Belgium). Automation in construction, 43, 1-9.

Tan, K., Cheng, X., Ju, Q., 2016. Combining mobile terrestrial laser scanning geometric and radiometric data to eliminate accessories in circular metro tunnels. Journal of applied remote sensing, 10(3), 030503.

Van Gosliga, R., Lindenbergh, R., Pfeifer, N., 2006. Deformation analysis of a bored tunnel by means of terrestrial laser scanning. na.

Walton, G., Delaloye, D., Diederichs, M. S., 2014. Development of an elliptical fitting algorithm to improve change detection capabilities with applications for deformation monitoring in circular tunnels and shafts. Tunnelling and Underground Space Technology, 43, 336-349.

Yi, C., Lu, D., Xie, Q., Liu, S., Li, H., Wei, M., Wang, J., 2019. Hierarchical tunnel modeling from 3D raw LiDAR point cloud. Computer-Aided Design, 114, 143-154. 